\title{
A Patient with Bacterial Bilateral Case Cervical Aneurysms Who Responded to Endovascular Treatment
}

Yoshifumi Tsuboi, ${ }^{1}$ Hiroshi Itokawa, ${ }^{2}$ Michihisa Narikiyo, ${ }^{1}$ Gota Nagayama, ${ }^{1}$ Hirokazu Nagasaki, ${ }^{1}$ Seiya Nagao, ${ }^{1}$ and Chisaku Kambayashi ${ }^{1}$

Objective: We report a case involving a patient in whom endovascular treatment for bilateral bacterial cervical aneurysms led to a favorable outcome.

Case Presentation: A 67-year-old male was diagnosed with bilateral bacterial cervical aneurysms during treatment for infectious endocarditis, and was referred to our hospital. Contrast-enhanced CT revealed aneurysms around the bifurcations of the bilateral carotid arteries. Antibiotic administration did not reduce the aneurysms, and endovascular treatment was performed. Stenting was conducted in the bilateral carotid arteries, and there was a marked reduction in the right aneurysm size. However, the left aneurysm was only transiently reduced and reappeared. Coil embolization was performed, leading to the disappearance of the left aneurysm.

Conclusion: Endovascular treatment may be an option for bacterial cervical aneurysms.

Keywords bacterial cervical aneurysm, stenting, coil embolization

\section{Introduction}

Bacterial aneurysms are associated with infection-related destruction of the arterial wall structure, and the incidence of rupture is higher than that observed in cases of noninfectious aneurysms. Therefore, surgery is required when antibiotic therapy does not reduce the aneurysm size. Direct surgery or endovascular treatment has reportedly yielded relatively favorable results when used for the treatment of bacterial intracranial aneurysms. ${ }^{1,2)}$ However, bacterial cervical aneurysms are difficult to treat., ${ }^{3,4)}$ This is because of the high risk of hemorrhage associated with

${ }^{1}$ Department of Neurosurgery, Neurovascular Center, Kawasakisaiwai Hospital, Kawasaki, Kanagawa, Japan

${ }^{2}$ Department of Neurosurgery, Shinyurigaoka General Hospital, Kawasaki, Kanagawa, Japan

Received: February 15, 2018; Accepted: April 21, 2018 Corresponding author: Yoshifumi Tsuboi. Department of Neurosurgery, Neurovascular Center, Kawasakisaiwai Hospital, 31-27 Omiyacho, Saiwai, Kawasaki, Kanagawa 212-0014, Japan

Email: tsuboyoshi@hotmail.com

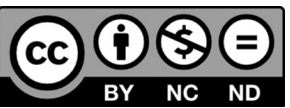

This work is licensed under a Creative Commons Attribution-NonCommercialNoDerivatives International License.

(C)2018 The Japanese Society for Neuroendovascular Therapy aneurysm-exfoliating operations, and the high probability of ischemic complications associated with angioplasty with a patch/graft or parent vessel occlusion.

In this study, we describe a case involving a patient in whom endovascular treatment for bilateral bacterial cervical aneurysms after implantation-type defibrillator infection led to a favorable course, and present a review of associated literature.

\section{Case Presentation}

A 67-year-old man presented to a hospital with consciousness disorder and fever. He had a medical history of hypertrophic cardiomyopathy and ventricular fibrillation. An implantation-type defibrillator was inserted 4 years prior to presentation. His inflammatory response was enhanced, and transthoracic echocardiography demonstrated verruca surrounding the tricuspid valve. A diagnosis of infectious endocarditis due to implantation-type defibrillator infection was made. On blood culture, Staphylococcus aureus (S. aureus) was detected, and intravenous vancomycin and cefazolin were started. The defibrillator was then removed. The inflammatory response was reduced 1 month after the initiation of treatment; however, swelling of the bilateral cervical arteries was observed. Contrast-enhanced CT 


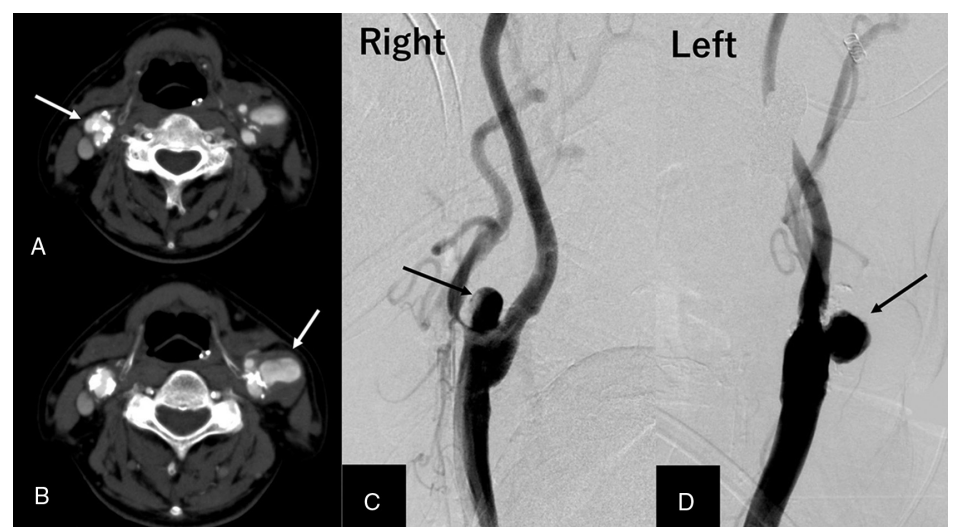

Fig. 1 Contrast-enhanced CT images (A and B) of aneurysms around the bifurcations of the left and right carotid arteries. Angiography revealed superior protrusion of the right aneurysm from the carotid artery bifurcation (C) and lateral protrusion of the left aneurysm from the common carotid artery (D).

revealed bilateral cervical aneurysms. Antibiotic therapy was continued, but the aneurysms did not shrink, and the patient was referred to our hospital 2 months after the initiation of treatment.

On physical examination, the patient was found to be conscious, but was unable to speak owing to tracheotomy. However, he was able to communicate by writing. There was no neurologic deficit. In the bilateral cervical arteries, pulsatile masses were observed.

Laboratory studies showed a leukocyte count and C-reactive protein level of $8430 / \mu \mathrm{L}$ and $0.5 \mathrm{mg} / \mathrm{dL}$, respectively, suggesting a reduction in the inflammatory response. Contrast-enhanced CT revealed an aneurysm measuring $12 \times 9 \mathrm{~mm}$ at the bifurcation of the right carotid artery and an aneurysm measuring $23 \times 22 \mathrm{~mm}$ at the bifurcation of the left carotid artery (Fig. 1A and 1B). Superior protrusion of the right aneurysm from the carotid artery bifurcation and lateral protrusion of the left aneurysm from the common carotid artery adjacent to the carotid artery bifurcation were observed via angiography (Fig. 1C and 1D).

Cefazolin, which had been prescribed at the previous hospital, was continuously administered. On blood culture, no bacteria were detected, and there was no fever after admission. There was no reduction in aneurysm size despite the reduction in inflammation 2 months after the start of treatment at the previous hospital; therefore, surgery was considered necessary. As bilateral lesions were present, minimally invasive endovascular treatment was selected. Initially, the insertion of foreign bodies, such as a coil, into the aneurysms was avoided, and stenting was adopted to potentially reduce aneurysm size.

As the patient had been taking aspirin, clopidogrel at a dose of $75 \mathrm{mg} /$ day was administered beginning 5 days before endovascular treatment. Initially, right carotid artery stenting was performed. A 6-Fr Shuttle Guiding Sheath (Cook Medical, Bloomington, IN, USA) was inserted into the right common carotid artery. A Guardwire (Medtronic, Minneapolis, MN, USA) was guided into the cervical portion of the right internal carotid artery. Under occlusion of the internal carotid artery, Carotid Wallstents (Boston Scientific, Natick, MA, USA) measuring $8 \times 29 \mathrm{~mm}$ and $8 \times$ $21 \mathrm{~mm}$, respectively, were inserted into the internal to common carotid arteries to overlap with each other at the aneurysm site. Using an Export, $40 \mathrm{~mL}$ of blood was aspirated, and there was no debris. Therefore, occlusion of the internal carotid artery was resolved. Angiography confirmed the intra-aneurysmal retention of contrast medium (Fig. 2A and 2B).

Left carotid artery stenting was performed 11 days later. A 6-Fr shuttle sheath was inserted into the left common carotid artery. A Guardwire was guided into the cervical portion of the left internal carotid artery to occlude the internal carotid artery. Carotid Wallstents measuring $10 \times$ $31 \mathrm{~mm}$ and $10 \times 24 \mathrm{~mm}$, respectively, were inserted into the internal to common carotid arteries to overlap with each other at the aneurysm site. Using an Export, $60 \mathrm{~mL}$ of blood was aspirated, and there was no debris. Therefore, occlusion of the internal carotid artery was resolved. Post-treatment angiography confirmed slight intra-aneurysmal retention of contrast medium (Fig. 2C and 2D).

Angiography 2 weeks after treatment demonstrated marked reduction in the bilateral aneurysms (Fig. 3A and $3 E$ ), and further reduction was confirmed 4 weeks after treatment (Fig. 3B and 3F). The modified Rankin Scale score was 3 , and the patient was referred to a recovery-phase rehabilitation hospital. 


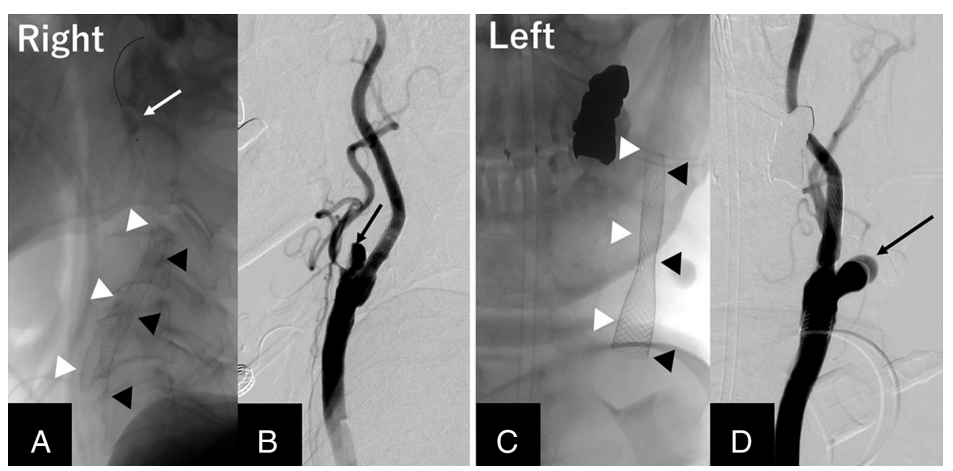

Fig. 2 (A) The right internal carotid artery was blocked using a Guardwire (Medtronic, Minneapolis, MN, USA) (arrow), and stenting involving the right internal to common carotid arteries was performed (first stent: white arrowheads, second stent: black arrowheads). After stenting, there was a decrease in the intra-aneurysmal influx of contrast medium (B). (C) Stenting involving the left internal to common carotid arteries was performed (first stent: white arrowheads, second stent: black arrowheads). After stenting, slight intra-aneurysmal retention of contrast medium was observed (D).

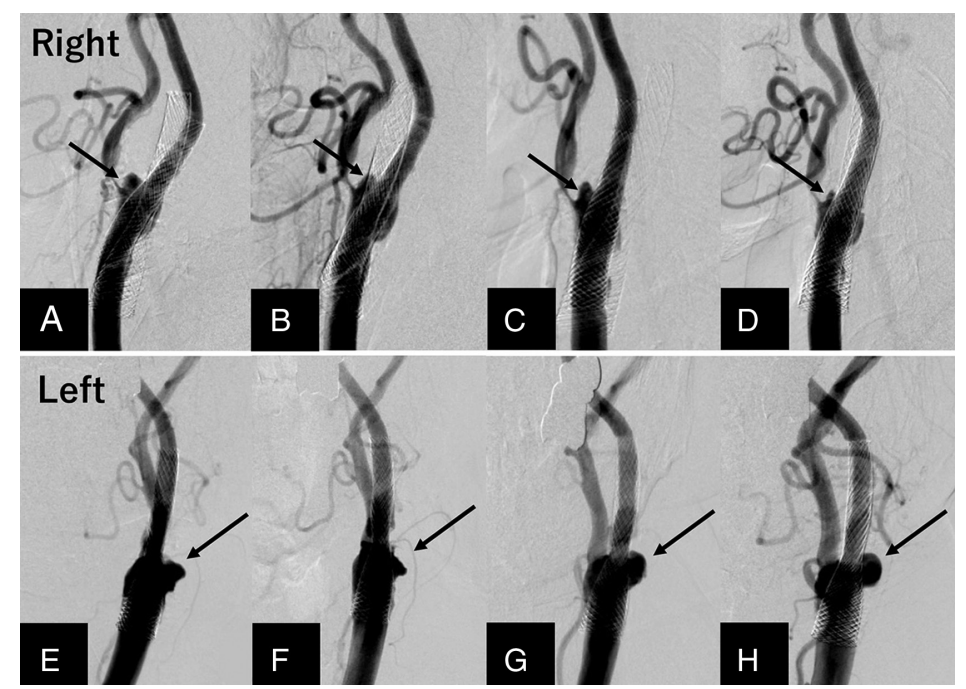

Fig. 3 Angiography 2 weeks after treatment ( $\mathbf{A}$ and $\mathbf{E}$ ) showed reduction in the left and right aneurysms. Angiography 4 weeks after treatment (B and $\mathbf{F}$ ) revealed further reduction in the aneurysms. Angiography 6 months after treatment ( $\mathbf{C}$ and $\mathbf{G}$ ) did not show any marked change in the right aneurysm, but slight enlargement of the left aneurysm was noted. Angiography 1 year after treatment ( $\mathbf{D}$ and $\mathbf{H}$ ) demonstrated a reduction in the right aneurysm, with a marked decrease in the contrast-enhanced area. However, further enlargement of the left aneurysm was observed.

No change in the visualization of the right aneurysm was noted on angiography 6 months after treatment in comparison with angiography performed 4 weeks after treatment. However, on the left side, the aneurysm reappeared (Fig. 3C and $\mathbf{3 G}$ ). At this point, clopidogrel, which was added prior to stenting, was discontinued, and aspirin alone was administered. However, on angiography 1 year after treatment, the left aneurysm was more clearly visualized (Fig. 3D and 3H). Therefore, coil embolization of the aneurysm was selected as an additional procedure.
A 9-Fr long sheath was inserted into the right femoral artery, and a 9-Fr Optimo (Tokai Medical Products, Aichi, Japan) was inserted into the left common carotid artery. As a back-up system for the embolization catheter, a 4.2-Fr FUBUKI (Asahi Intecc Co., Ltd., Aichi, Japan) was inserted to an area adjacent to the aneurysmal orifice. Using a 200-cm CHIKAI 0.014 (Asahi Intecc Co., Ltd.), an Echelon 10 (MTI-ev3, Irvine, CA, USA) was guided into the aneurysm through the stent mesh overlapped during the previous treatment session (Fig. 4A). Two $6 \mathrm{~mm} \times 20 \mathrm{~cm}$ 


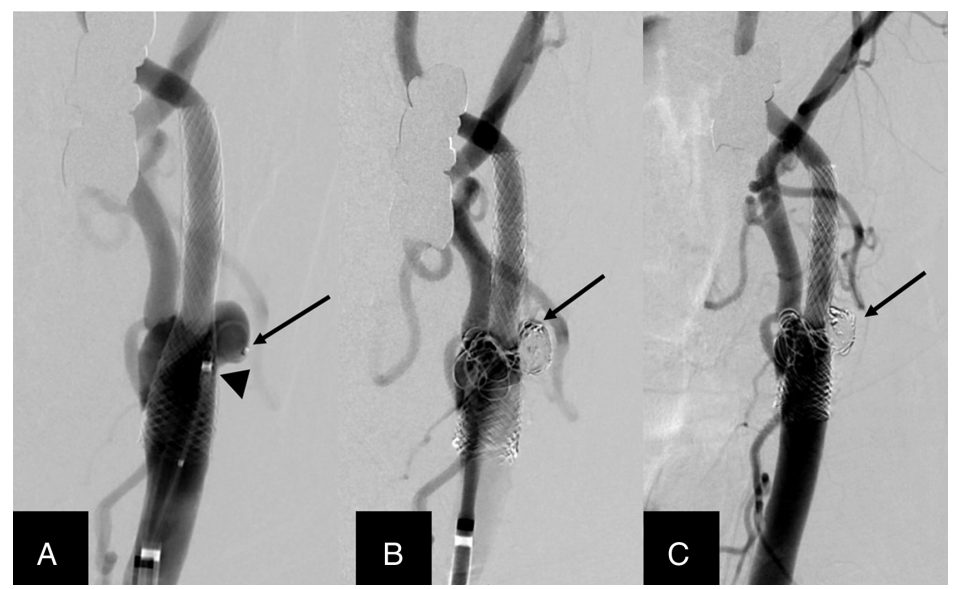

Fig. 4 (A) A 4.2-Fr FUBUKI (Asahi Intecc Co., Ltd., Aichi, Japan) was inserted to an area adjacent to the aneurysm (arrowhead), and an Echelon 10 (MTI-ev3, Irvine, CA, USA) was guided into the aneurysm (arrow). After coil insertion (B), slight influx of contrast medium in the superior area of the aneurysm (arrow) was noted. On angiography 6 months after coil embolization (C), there was no visualization of the left aneurysm.

Target 360 soft coils (Stryker, Kalamazoo, MI, USA), one $5 \mathrm{~mm} \times 20 \mathrm{~cm}$ Target $360 \mathrm{soft}$ coil, two $4 \mathrm{~mm} \times 8 \mathrm{~cm}$ Target 360 soft coils, and two $3 \mathrm{~mm} \times 8 \mathrm{~cm}$ Target 360 soft coils were inserted. In the superior area of the aneurysm, slight influx of contrast medium was observed (Fig. 4B), but microcatheter deviation into the parent blood vessel made re-insertion impossible. Treatment was then completed. On angiography 6 months after treatment, there was no visualization of the left aneurysm (Fig. 4C). One year after coil embolization, the tracheotomy site was closed. Cervical swelling disappeared, and there have been no sequelae.

\section{Discussion}

Bacterial cervical aneurysms are rare. According to a review of 74 patients with bacterial extracranial carotid artery aneurysms, published by Knouse et al., the most frequent causative bacteria were S. aureus, followed by enteric bacteria, such as Salmonella and Escherichia coli, and Streptococcus. ${ }^{3)}$ Furthermore, Nakamura et al.5) reported that etiological factors for infection included surgery, sepsis, infectious endocarditis, and peritonsillar infection, and indicated that there was a bimodal distribution, with peaks at 10-19 years and 60-69 years of age.

Two mechanisms may be involved in the formation of bacterial aneurysms. First, sepsis-related bacterial implantation in the vascular intima may weaken the vascular wall, leading to the formation of aneurysms. The other mechanism is that an infected focus of the periarterial tissue may involve the arterial wall, thereby destroying it. ${ }^{6)}$ In the present case, infectious endocarditis was present, suggesting the former mechanism. Concerning the site of bacterial aneurysms, a study found that bacteria frequently adhered to arteriosclerotic lesions. ${ }^{5)}$ In the present case, slight stenosis was noted at areas proximal to the bilateral aneurysms; therefore, the bacteria may have adhered to the site of arteriosclerosis, leading to the formation of the aneurysms.

As reported for bacterial intracranial aneurysms, antibiotic therapy is also predominantly selected in the treatment of bacterial cervical aneurysms. However, according to one study, the absence of treatment for the residual aneurysm caused aneurysmal rupture or embolic cerebral infarction with a mortality rate of $70 \%-90 \%{ }^{4}{ }^{4}$ Therefore, if antibiotic therapy does not reduce the aneurysm size, surgery should be promptly performed. Surgical procedures for bacterial cervical aneurysms include ligation of the carotid artery, ${ }^{7,8)}$ aneurysm resection/patch closure, and revascularization with an artificial blood vessel or saphenous vein graft. ${ }^{9-12)}$ Concerning ligation of the carotid artery, it was reported that the incidence of postoperative stroke was 50\%-60\%, ${ }^{3)}$ and that mortality ranged from $17 \%$ to $40 \%$. $^{4}$ Thus, ligation of the carotid artery should be indicated only for patients in whom revascularization is impossible. However, previous studies demonstrated that the mortality rate was $10 \%-20 \%$ even when performing aneurysm resection and revascularization. ${ }^{3,4)}$ Overall, these procedures may cause ischemic complications.

The present case demonstrated a favorable treatment course. As shown in Table 1, eight case reports on endovascular treatment for bacterial cervical aneurysms have 


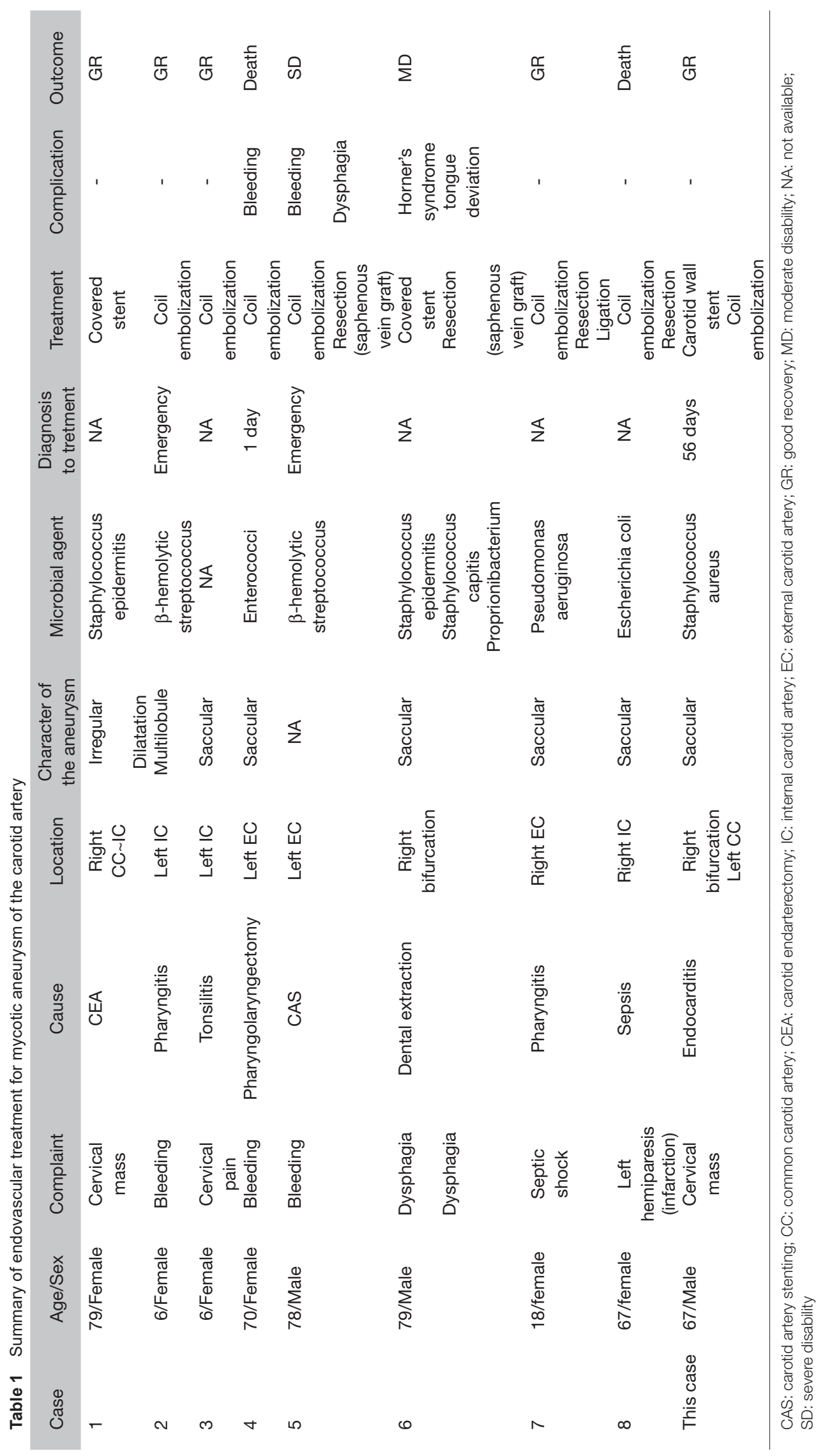


been published to date. ${ }^{12-19)}$ Etiological factors for aneurysms included surgery/treatment in four patients, peripharyngeal inflammation in three patients, and sepsis in one patient. Concerning the characteristics of aneurysms, irregular dilation was observed in one patient, multilobular aneurysm in one patient, and saccular aneurysms in six patients. Of these, endovascular treatment was performed for four patients (Cases 1, 2, 3, and 4) to achieve radical cure. For the remaining four patients, stenting or coil embolization was performed as pretreatment prior to direct surgery. Among the eight patients, there was no exacerbation of infection after endovascular treatment. Stenting led to radical cure in one patient (Case 1); to treat the irregular aneurysm, a covered stent was inserted, resulting in a marked reduction in the aneurysm. To achieve radical cure, coil embolization was performed for three patients (Cases 2, 3, and 4). In Cases 2 and 3, after evaluating a collateral pathway, coil embolization of the aneurysmal base to the parent blood vessel was performed, leading to a favorable outcome. In Case 4, coil migration induced additional hemorrhage, resulting in an unfavorable outcome. In Case 5, microhemorrhage of the disintegrated skin persisted from embolization of the aneurysm until direct surgery. In Cases 4 and 5, the aneurysms were adjacent to the bifurcation of the carotid artery, and the extent of parent artery occlusion was limited. When conducting coil embolization, coil migration may occur; therefore, after evaluating a collateral pathway, a parent artery should be sufficiently embolized.

In the present case, after confirming a negative inflammatory response related to antibiotic therapy and the absence of bacteria on blood culture, we selected minimally invasive stenting due to the presence of bilateral lesions. Stenting can be performed relatively safely if a stent is inserted under adequate protection considering the risk of embolic complications. On the other hand, we cannot rule out the possibility that insertion of a foreign body exacerbates bacterial aneurysms. Therefore, this procedure should be performed after the reduction of infection. Regarding exacerbation of infection, preparation for prompt direct surgery may be important.

For carotid artery stents, open-cell stents, such as a Precise Pro RX (Cordis, Miami, FL, USA) and PROTÉGÉ RX (Covidien Irvine, CA, USA), and closed-cell stents, such as a Carotid Wallstent, are used. In the present case, we used a Carotid Wallstent with a smaller free cell area to obtain improvement of the aneurysm. However, if flexion of the carotid artery is marked, a stent may not crimp to a blood vessel; therefore, the use of an open-cell stent must be considered.

In the present case, stenting promptly reduced the right aneurysm, but there was an increase in the contrastenhanced area of the left aneurysm, and coil embolization was performed. Enlargement of the left aneurysm despite reduction in the right aneurysm may have been associated with the site of development. The right aneurysm developed at the bifurcation of the carotid artery, whereas the left aneurysm developed in the common carotid artery. In the common carotid artery, the stent cell area was larger than at the bifurcation, which may have reduced the therapeutic effects. Furthermore, coil embolization may exacerbate infection, as indicated for stenting. In the present case, follow-up was continued for $\geq 1$ year after stenting; therefore, the risk of coil-insertion-related recrudescence of infection was considered to be low.

Currently, we cannot conclude whether endovascular treatment is effective. However, it is minimally invasive, suggesting its usefulness as pretreatment before direct surgery instead of radical surgery. Endovascular treatment may be an option for bacterial cervical aneurysms.

\section{Disclosure Statement}

The authors declare that they have no conflict of interest.

\section{References}

1) Chun JY, Smith W, Halbach VV, et al: Current multimodality management of infectious intracranial aneurysms. Neurosurgery 2001; 48: 1203-1213; discussion 1213-1214.

2) Park W, Ahn JS, Park JC, et al: Treatment strategy based on experience of treating intracranial infectious aneurysms. World Neurosurg 2017; 97: 351-359.

3) Knouse MC, Madeira RG, Celani VJ: Pseudomonas aeruginosa causing a right carotid artery mycotic aneurysm after a dental extraction procedure. Mayo Clin Proc 2002; 77: 1125-1130.

4) Pirvu A, Bouchet C, Garibotti FM, et al: Mycotic aneurysm of the internal carotid artery. Ann Vasc Surg 2013; 27: 826-830.

5) Nakamura T, Toyama K, Sato S, et al: [A case of mycotic pseudoaneurysm of the external carotid artery following a peritonsillar abscess]. Nippon Jibiinkoka Gakkai Kaiho 2014; 117: 122-127. (in Japanese)

6) Rogers AC, Bourke M, Galbraith AS, et al: Mycotic aneurysm of the extracranial internal carotid artery, resect and ligate or reconstruct? Ann Vasc Surg 2016; 35: 203.e5-203.e10. 
7) Lambert MJ, Johns ME, Mentzer R, et al: Mycotic carotid artery aneurysm. Otolaryngol Head Neck Surg 1979; 87: 624-627.

8) Lueg EA, Awerbuck D, Forte V: Ligation of the common carotid artery for the management of a mycotic pseudoaneurysm of an extracranial internal carotid artery. A case report and review of the literature. Int J Pediatr Otorhinolaryngol 1995; 33: 67-74.

9) Chan YC, Cheng SW: Mycotic aneurysm of the common carotid artery as a presenting symptom for early colorectal malignancy. Ann Vasc Surg 2016; 30: 306.e9-306.e12.

10) Kuy S, Dua A, Desai SS, et al: Ruptured mycobacterial aneurysm of the carotid artery. Perspect Vasc Surg Endovasc Ther 2013; 25: 53-56.

11) Naik DK, Atkinson NR, Field PL, et al: Mycotic cervical carotid aneurysm. Aust N Z J Surg 1995; 65: 620-621.

12) Kaviani A, Ouriel K, Kashyap VS: Infected carotid pseudoaneurysm and carotid-cutaneous fistula as a late complication of carotid artery stenting. J Vasc Surg 2006; 43: 379-382.

13) Baril DT, Ellozy SH, Carroccio A, et al: Endovascular repair of an infected carotid artery pseudoaneurysm. $J$ Vasc Surg 2004; 40: 1024-1027.
14) Brochu B, Dubois J, Garel L, et al: Complications of ENT infections: pseudoaneurysm of the internal carotid artery. Pediatr Radiol 2004; 34: 417-420.

15) Chamseddin KH, Kirkwood ML: Lemierre's syndrome associated mycotic aneurysm of the external carotid artery with primary internal carotid artery occlusion in a previously healthy 18-year-old female. Ann Vasc Surg 2016; 36: 291.e11-291.e14.

16) Goddard AJ, Lenthall RK, Bradley PJ: Endovascular management of infected carotid artery pseudoaneurysm complicating pharyngolaryngectomy: complete occlusion followed by early recurrence and rebleeding. J Laryngol Otol 2004; 118: 991-995.

17) Reisner A, Marshall GS, Bryant K, et al: Endovascular occlusion of a carotid pseudoaneurysm complicating deep neck space infection in a child. Case report. $J$ Neurosurg 1999; 91: 510-514.

18) Tsai TC, Barot N, Dalman R, et al: Combined endovascular and open operative approach for mycotic carotid aneurysm. J Vasc Surg 2010; 51: 1514-1516.

19) Wales L, Kruger AJ, Jenkins JS, et al: Mycotic carotid pseudoaneurysm: staged endovascular and surgical repair. Eur J Vasc Endovasc Surg 2010; 39: 23-25. 